\title{
Identification of HIVEP2 as a dopaminergic transcription factor related to substance use disorders in rats and humans
}

\author{
Juan Zhao ${ }^{1,2}$, Chunnuan Chen ${ }^{2,3}$, Richard L. Bell ${ }^{4}$, Hong Qing (1) ${ }^{1}$ and Zhicheng Lin ${ }^{2}$
}

\begin{abstract}
Playing an important role in the etiology of substance use disorder (SUD), dopamine (DA) neurons are subject to various regulations but transcriptional regulations are largely understudied. For the first time, we report here that the Human Immunodeficiency Virus Type I Enhancer Binding Protein 2 (HIVEP2) is a dopaminergic transcriptional regulator. HIVEP2 is expressed in both the cytoplasm and nuclei of DA neurons. Therein, HIVEP2 can target the intronic sequence GTGGCTITCT of SLC6A3 and thereby activate the gene. In naive rats from the bi-directional selectively bred substance-preferring P vs -nonpreferring NP rat model of substance abuse vulnerability, increased gene activity in males was associated with the vulnerability, whereas decreased gene activity in the females was associated with the same vulnerability. In clinical subjects, extensive and significant HIVEP2-SLC6A3 interactions were observed for SUD. Collectively, HIVEP2-mediated transcriptional mechanisms are implicated in dopaminergic pathophysiology of SUD.
\end{abstract}

\section{Introduction}

Many environmental factors can regulate dopaminergic (DAergic) activity including SLC6A3, the gene encoding the dopamine transporter $(\mathrm{DAT})^{1-7}$. These regulations remain mechanistically poorly understood. A critical step in dissecting these mechanisms is to identify what transcription factors (TFs) target the DAergic genome. Recently, several TFs for SLC6A3 have been cloned, including Nurr1 (NR4A2), paired-like homeobox 3 protein (PITX3), HEY1, SP1, SP3, ${ }^{\mathrm{AZI}} 3^{\prime}$ UTR, SRP54, and $\mathrm{Nfe} 2 \mathrm{l}^{8-13}$. It is postulated that HEY1 is a TF targeting the $3^{\prime} \mathrm{UTR}^{14}$, whereas the others appear to target $5^{\prime}$ promoter regions except ${ }^{\mathrm{AZI}} \mathrm{3}^{\prime} \mathrm{UTR}$, a long noncoding RNA (lncRNA), that regulates Intron 1 of SLC6A3. Among these TFs, Nurr1, but not PITX3, is implicated in Parkinson's disease ${ }^{15,16}$ and ${ }^{\mathrm{AZI}}{ }^{\prime}{ }^{\prime}$ UTR is implicated in substance use disorder (SUD $)^{13}$. Importantly, both diseases

\footnotetext{
Correspondence: Zhicheng Lin (zlin@mclean.harvard.edu)

'School of Life Science, Beijing Institute of Technology, 100081 Beijing, China 'Laboratory of Psychiatric Genomics, McLean Hospital, Belmont, MA 02478, USA

Full list of author information is available at the end of the article.
}

are closely associated with altered DAergic activity. These findings indicate that identification of DAergic TFs may provide opportunities for dissecting signaling pathways that target these TFs for genomic action. This would help clarify molecular cascades underlying neuronal development, plasticity and DAergic transcription-related disorders such as SUD ${ }^{17-20}$.

The present study focused on delineation of new transcription mechanisms in SUD, using SLC6A3 as a prototype target to clone DAergic TFs. Previous studies, including ours, have shown that a 121-bp fragment in Intron 1 of $S L C 6 A 3$ may display cis-acting activity in vitro. This 121-bp fragment served as the bait/target in our search for new DAergic TFs related to SUD.

\section{Materials and methods}

All DNA or RNA oligonucleotides used in this study are shown in Table 1.

\section{Cloning of TFs via Yeast One Hybrid System $(\mathrm{Y} 1 \mathrm{H})^{21}$}

The 121-bp fragment of haplotype $\mathrm{A}^{22}$ was used as the search target and a human adult brain cDNA library was 
Table 1 Oligonucleotide List (DNA and RNA)

\begin{tabular}{|c|c|c|}
\hline Name & Sequence & $A C$ \\
\hline \multicolumn{3}{|c|}{ a. cDNA cloning (Figs. 3, 4b) } \\
\hline HIVEP2 forward & 5'-aaaaagctagcaacaaaatggacactggggacaca-3' & \\
\hline HIVEP2 reverse & $5^{\prime}$-aaaaagcggccgcatcaatgtagctgactcttttctgat-3' & \\
\hline \multicolumn{3}{|c|}{ b. Plasmid construction (Fig. 4b,d) } \\
\hline 1-forward & 5'-CTA GAG AAA GCC ACG-3' & \\
\hline 1-reverse & 5'-TCG ACG TGG CTT TCT-3' & \\
\hline 2A-forward & 5'-CTA GGG AGA GGC AAA-3' & \\
\hline 2A-reverse & 5'-TCG ATT TGC CTC TCC-3' & \\
\hline 2B-forward & 5'-CTA GGG AGG AGC AAA-3' & \\
\hline 2B-reverse & 5'-TCG ATT TGC TCC TCC-3' & \\
\hline \multicolumn{3}{|c|}{ c. HIVEP2 DsiRNA (Fig. 4c) } \\
\hline Sense & 5'-GGUCUGGAGAAACUGAUAAAGCATC-3' & \\
\hline Antisense & 5'-GAUGCUUUAUCAGUUUCUCCAGACCUU -3' & \\
\hline \multicolumn{3}{|c|}{ d. qRT-PCR primers (Figs. 3b-d, 5) } \\
\hline SLC6A3 forward & 5'-ATC TAT GCG GCC TAC AAG TT-3' & 2.002 \\
\hline SLC6A3 reverse & 5'-CTC TAC ACC TTG AGC CAG T-3' & \\
\hline $\begin{array}{l}\text { hGAPDH } \\
\text { forward }\end{array}$ & 5'-AGG CCG GAT GTG TTC G-3' & 2.017 \\
\hline $\begin{array}{l}\text { hGAPDH } \\
\text { reverse }\end{array}$ & $5^{\prime}-T$ A CCA GAG TTA AAA GCA GCC-3' & \\
\hline rHIVEP2 forward & $5^{\prime}-\mathrm{CTT}$ CCT GTC CAC CTC ACT T-3' & 2.027 \\
\hline rHIVEP2 reverse & 5'-CCA GAC CTG ACC TCG TCT A-3' & \\
\hline rDAT forward & 5'-TCA CTC TTG GCA TTG TCC TG-3' & 1.972 \\
\hline rDAT reverse & $5^{\prime}-\mathrm{GAG}$ CAC GCC AAA GAG GAT AG-3' & \\
\hline $\begin{array}{l}\text { rGAPDH } \\
\text { forward }\end{array}$ & 5'-ATG ACT CTA CCC ACG GCA AG-3' & 2.034 \\
\hline rGAPDH reverse & 5'-TAC TCA GCA CCA GCA TCA CC-3' & \\
\hline \multicolumn{3}{|c|}{ e. ChIP-PCR (Fig. 2) } \\
\hline $\begin{array}{l}\text { HIVEP2-DNPi } \\
\text { forward }\end{array}$ & 5'-ACA TTA TTG AAT GCT CTT AGA AG-3' & \\
\hline $\begin{array}{l}\text { HIVEP2-DNPi } \\
\text { reverse }\end{array}$ & 5'-GCC TCA AGA CAG ACA CTC T-3' & \\
\hline $\begin{array}{l}\mathrm{hDAT}+0.79 \mathrm{~kb} \\
\text { forward }\end{array}$ & 5'-TCG TCG GGT GTT TTA CCC AC-3' & \\
\hline $\begin{array}{l}\mathrm{hDAT}+0.79 \mathrm{~kb} \\
\text { reverse }\end{array}$ & 5'-GTG GAG GCT CTA ACA GGC AA-3' & \\
\hline hACTB forward & 5'-AAA GGC AAA CAC TGG TCG GA-3' & \\
\hline hACTB reverse & 5'-GGG ACT CAA GGC GCT AAC TG-3' & \\
\hline
\end{tabular}

constructed into pP6, a pGADGH derivative plasmid, both used in $\mathrm{Y} 1 \mathrm{H}$ in the search for proteins targeting the 121-bp (Hybrigenics Services, Paris, France). The bait construct was first checked by re-sequencing and then transformed into the yeast strain Y1H300 (MAT a, Gal4 $\Delta$, ade2-101, trp1-901, leu2-3,112, his3 $\Delta 200$ ), integrating the DNA bait into the yeast genome. Screening was performed against the random-primed cDNA library. Clones were screened and complete cDNAs were cloned as described before ${ }^{12}$.

\section{Plasmid construction}

Human HIVEP2 cDNA ORF $(7.3 \mathrm{~kb})$ was cloned by PfuUltra High-fidelity DNA Polymerase (Agilent Technologies) chain reaction (PCR) and then ligating the PCR product into the indicated sites in the mammalian expression vector pcDNA3.1+, resulting in the plasmid pcDNA3.1 + HIVEP2. The cloning PCR used primers for NheI (NEB)/NotI (NEB) sites-directed insertion. The PCR templates were cDNAs synthesized from total RNAs isolated from a combination of human SK-N-AS, IMR-32, and SH-SH5Y cells ${ }^{23}$. PCR fidelity was verified by DNA re-sequencing.

For luciferase (Luc) assay ${ }^{13}$, three reporter plasmids were generated: $1,2 \mathrm{~A}$ and $2 \mathrm{~B}$. For constructing each plasmid, two complementary single-stranded synthetic oligonucleotides were annealed together into a doublestranded oligonucleotide with NheI at the $5^{\prime}$ end and XhoI (NEB) at the $3^{\prime}$ end, followed by ligation of the doublestranded oligonucleotide to the same restriction sites immediately upstream of the SV40 promoter in Promega's pGL3 Promoter Vector.

\section{Cell culture and transfection}

SK-N-AS and BE(2)-M17 cell lines were purchased from ATCC and maintained in Dulbecco's modified eagle's medium (DMEM, Gibco) containing 10\% fetal bovine serum (FBS, Atlanta Biologicals) and 1\% Pen/Strep (Gibco). One percent non-essential amino acids (Hyclone) were added to medium for SK-N-AS. Cells were incubated at $37{ }^{\circ} \mathrm{C}$ in humidified air with $5 \% \mathrm{CO}_{2}$. Cell lines were verified by PCR genotyping and tested for or removed of mycoplasma contamination (Biotool Mycoplasma Removal Kit, Bimake).

Cells were transfected chemically with plasmid DNA in Polyethylenimine (PEI, Polysciences) or lipfectamine2000. Plasmid DNA and PEI were diluted in DMEM without serum separately. The PEI solution was then added into the DNA solution, followed by vortexing for $10 \mathrm{~s}$ and incubation at room temperature (RT) for $15 \mathrm{~min}$ to form DNA-PEI complexes. At the end, the complexes were added to each well containing fresh $2 \%$ FBS growth medium. Medium was changed into fresh 10\% FBS complete growth medium the next day. For 2-hexyl-4pentynoic acid (HPA, ApexBio) treatment, HPA was added into cell medium $2 \mathrm{~h}$ after transfection at $5 \mu \mathrm{M}$ / well. Sixteen hours after transfection, cells were collected 
for quantitative reverse transcription PCR (qRT-PCR). Three separate experiments were performed for statistical analysis at least.

\section{Animals}

All animals, rats (alcohol preferring or P rat and alcohol non-preferring or NP rat, generation 76 and 79 from Indiana University) or male mice (C57BL/6NTac, from Taconic Biosciences, Rensselaer, New York, USA), were all adults (2-4 month old) housed under constant temperature- $\left(21^{\circ} \mathrm{C}\right)$ and humidity- $(50 \%)$ on a $12 \mathrm{~h} / 12 \mathrm{~h}$ light-dark cycle (light 07:00-19:00) with food and water available ad libitum. Rodents were housed in plastic ventilated cages. Mice housed up to five per cage, and rats housed up to two per cage. No animal was housed singly. Four individuals were used in each experiment for statistical analysis at least. All experimental procedures were approved by the Institutional Animal Care and Use Committees of McLean Hospital or the Indiana University Schools of Dentistry and Medicine (Indianapolis, IN) and were in accordance with the guidelines of the Institutional Animal Care and Use Committee of the National Institute on Drug Abuse, National Institutes of Health, and the Guide for the Care and Use of Laboratory Animals. Indicated sample size was determined by gene activity stability without randomization/blinding involved for molecular analyses.

\section{Immunofluorescent staining (IFS) of cells and rodent brain sections}

Rabbit anti-HIVEP2 sera were kindly provided by Shunsuke Ishii of Institute of Physical and Chemical Research (RIKEN) in Japan. Methods were as described before $^{12}$. For SK-N-AS cell staining, coverslips were blocked in 7\% non-fat milk at RT for $1 \mathrm{~h}$. Coverslips were incubated with rabbit ant-HIVEP2 sera (1:50 diluted in $0.01 \%$ BSA in Tris buffered saline (TBS)) or with diluted normal rabbit serum (Gibson Bioscience) as control at $4{ }^{\circ} \mathrm{C}$ for $48 \mathrm{~h}$. The secondary antibody is Alexa Fluor 568 goat anti-rabbit IgG (1:500 diluted, RRID AB_143157, Invitrogen). Images were obtained by confocal laser scanning microscopy (Leica TCS SP8).

For mouse or rat brain section staining, adult animals were anaesthetized with $250 \mathrm{mg} / \mathrm{kg}$ Avertin (Acros Organics) by intraperitoneal (i.p.) injection. Brains were promptly collected and frozen. Before slicing, the brain was fixed in $4 \%$ PFA at $4{ }^{\circ} \mathrm{C}$ for $24 \mathrm{~h}$ and was cryoprotected in $15 \%(\mathrm{wt} / \mathrm{v})$ sucrose overnight and then in $30 \%$ (wt/v) sucrose until the brain sank. After being embedded in optimal cutting temperature (OCT) compound, the frozen brain was coronally sectioned at $20 \mu \mathrm{m}$ on a cryostat (Thermo, Microm HM 505 E). Coronal sections containing the substantia nigra and the ventral tegmental area (VTA) were selected and washed three times, $5 \mathrm{~min}$ each, with $0.01 \mathrm{M}$ PBS for free floating staining. The sections were incubated with $0.3 \%$ Triton X-100 (diluted in PBS) for $30 \mathrm{~min}$ first, from the blocking step, the treatments were the same as those for cells, using mouse monoclonal anti-Tyrosine Hydroxylase (TH, 1:800 diluted in TBS containing $0.01 \%$ BSA, Millipore) for a marker of dopaminergic neurons.

\section{Western blotting}

Western blot analyses were performed on protein fractions from cultured cells, or VTA of adult mice and rats as method described before ${ }^{13}$. In HIVEP2 siRNA experiment, cells were transfected with lipfectamine2000 according to the manufacturer's instructions. 50pmol DsiRNA was applied in each well. Cells were lysed $48 \mathrm{~h}$ after transfection by adding $100 \mu \mathrm{L} /$ well of RIPA buffer (Gendepot) supplemented with protease inhibitor cocktail (Millipore). Five cycles of sonication ( $5 \mathrm{~s}$ on and $5 \mathrm{~s}$ off) were applied for nuclear protein. Protein concentration was determined by BCA (Bicinchoninic acid) kit (Thermo Scientific) according to the manufacturer's instruction.

For rodents, adult animals were killed by decapitation, brains were promptly collected and dissected in metal matrix (RWD). Two millimeters thick coronal brain section containing VTA between matrix 19 and $21 \mathrm{~mm}$ (bregma -4.52 to $-6.8 \mathrm{~mm}$ ) was cut and collected for rat brains; $1 \mathrm{~mm}$ thick coronal brain section between matrix 8 and $9 \mathrm{~mm}$ (bregma -2.92 to $-3.88 \mathrm{~mm}$ ) was cut-collected for mouse brains. VTA was sliced out of the section at one third from ventral side in the horizontal plane by removing overlying cortex and hippocampus at the periphery.

Cytoplasm and nuclear proteins of the VTA were separated using Lysis Buffer J from Cytoplasmic \& Nuclear RNA purification Kit (Norgen Biotek) according to the manufacturer's instructions. Nuclei were pelleted $\left(4{ }^{\circ} \mathrm{C}, 12,000 \times g\right.$ for $\left.10 \mathrm{~min}\right)$ and washed three times with cold PBS while the supernatants were collected as cytoplasm protein samples. RIPA buffer supplemented with protease inhibitor cocktail was used to lyse the nuclei as described above, the supernatants were collected as nuclear protein fractions.

Fifty $\mu \mathrm{g}$ total protein per loading well was electrophoretically separated on a $4-15 \%$ Criterion TGX precast gel (Bio-Rad). Polyvinylidene difluoride (PVDF) membranes (Santa Cruz) were incubated with primary antibody at $4{ }^{\circ} \mathrm{C}$ for two days. Primary antibodies used for western blots were as follows: rabbit anti-HIVEP2 sera (dilution at 1:1000), mouse anti-glyceraldehyde-3phosphate dehydrogenase (GAPDH, 1:10,000, RRID AB_10613283, Biolegend, for cells), mouse anti-GAPDH (1:200, RRID AB_2107299, Santa Cruz, for rat brain), goat anti-RNA Pol II (1:100, RRID AB_2167471, Santa Cruz, for nuclear protein control), mouse anti-TH (1:1000, 
RRID AB_2201528, Millipore, for cytoplasm protein control). Amersham ECL Western Blotting Analysis System (GE Healthcare) was used to detect the primary antibody activity according to the manufacturer's instruction. The images were captured by the Bio-Rad Molecular Imager (Bio-Rad, ChemiDOCIM XRS+) and quantitative assessment of protein bands, using the area under curve method, was executed by the Image J Software (NIH).

\section{Quantitative reverse transcription PCR (qRT-PCR)}

For cells and rat tissues, RNA were extracted by TRIzol (Ambion), following the manufacturer's protocol. RNA concentration was estimated with NanoDrop Lit (Thermo Fisher Scientific). Two hundred nanograms of total RNAs was reverse transcribed into cDNA by using the Verso cDNA synthesis kit (Thermo scientific) with oligo dT primers following the manufacturer's protocol. cDNA was diluted by 10 fold with DNase-free water. cDNA samples were amplified in triplicates by incubation in the Bio-Rad CFX Connect real-time system. The amplification condition was $95^{\circ} \mathrm{C}$ for $5 \mathrm{~min}$, then for 49 cycles of $95^{\circ} \mathrm{C}$ for $15 \mathrm{~s}, 55^{\circ} \mathrm{C}$ for $20 \mathrm{~s}$ and $72^{\circ} \mathrm{C}$ for $30 \mathrm{~s}$ using SsoAdvanced Universal SYBR green supermix (Bio-Rad) in a final volume of $12.5 \mu \mathrm{L}$, containing $1 \mu \mathrm{L}$ of cDNA and a final concentration of $0.5 \mu \mathrm{M}$ of forward and reverse primers. The gene of interest was then normalized against the reference gene GAPDH. The relative expression of the target gene was calculated according to the method as previously described ${ }^{12}$.

\section{Luc activity assay}

Forty-eight hours after HIVEP2 overexpression or DsiRNA co-transfected with reporter constructs, cell lysates were collected for Luc activity assay and protein concentration measurements. Luc activities were measured by Promega's Luciferase Assay System with BioTek/Gen5 (Winooski). Protein assays were performed with Coomassie blue according to the manufacturer's instructions (Thermoscientific). Briefly, $250 \mu \mathrm{L}$ of Coomassie protein assay reagent was added to $5 \mu \mathrm{L}$ of lysates and BSA standards. Luc activity value to protein concentration ratios were calculated for statistical analysis. Each assay was performed in duplicate.

\section{Chromatin immunoprecipitation (ChIP) PCR and qPCR}

ChIP method was described as before ${ }^{12}$. Around $5 \times$ $10^{6} \mathrm{BE}(2)$-M17 cells or $100 \mathrm{mg}$ of human brain tissue were collected for ChIP assay. Tissue in $200 \mu \mathrm{L} 1 \%$ formaldehyde was homogenized with a pestle mixer (Argos) and crosslinked at RT for $15 \mathrm{~min}$. Independently, cells were crosslinked with $1 \%$ formaldehyde at $\mathrm{RT}$ for $10 \mathrm{~min}$. The lysate was sonicated 12 cycles for BE (2)-M17 cells or 24 cycles for brain tissues (30 s on and
$30 \mathrm{~s}$ off). Supernatants were transferred into new tubes and diluted 1:4 with Dilution Buffer (0.01\% SDS, 1.1\% Triton X-100, $1.2 \mathrm{mM}$ EDTA, $16.7 \mathrm{mM}$ Tris-HCl, $\mathrm{pH}$ 8.0, $167 \mathrm{mM} \mathrm{NaCl}$ plus the protease inhibitors) and precleared with $25 \mu \mathrm{L}$ Dynabeads Protein A (Invitrogen) at $4{ }^{\circ} \mathrm{C}$ for $2 \mathrm{~h}$ with gentle rotation. The cleared supernatant was divided into two tubes and each incubated with $10 \mu \mathrm{L}$ anti-HIVEP2 sera or control rabbit serum at $4{ }^{\circ} \mathrm{C}$ for $48 \mathrm{~h}$ and then immunoprecipitated with $25 \mu \mathrm{L}$ Protein A beads on a rotator at $4{ }^{\circ} \mathrm{C}$ for $4 \mathrm{~h}$. Five hundred microliters of supernatant from the tube incubated with control rabbit IgG was collected as Input. The immunoprecipitates were washed sequentially with the following buffers: low salt buffer $(0.1 \%$ SDS, $1 \%$ Triton $\mathrm{X}-100,2 \mathrm{mM}$ EDTA, $20 \mathrm{mM}$ Tris- $\mathrm{HCl} \mathrm{pH}$ 8.0, $150 \mathrm{mM}$ $\mathrm{NaCl})$, high salt buffer $(0.1 \%$ SDS, $1 \%$ Triton $\mathrm{X}-100$, $2 \mathrm{mM}$ EDTA, $20 \mathrm{mM}$ Tris- $\mathrm{HCl} \mathrm{pH}$ 8.0, $500 \mathrm{mM} \mathrm{NaCl}$ ), $\mathrm{LiCl}$ wash buffer $(0.25 \mathrm{M} \mathrm{LiCl}, 1 \%$ Sodium deoxycholate, $1 \mathrm{mM}$ EDTA, $10 \mathrm{mM}$ Tris- $\mathrm{HCl}, \mathrm{pH} 8.0)$, and TE (10 mM Tris- $\mathrm{HCl}, 1 \mathrm{mM}$ EDTA, pH8.0). DNA was then eluted with $500 \mu \mathrm{L}$ of elution buffer (1\% SDS, $0.1 \mathrm{M} \mathrm{NaHCO}_{3}$ ) and the crosslinking was reversed by adding $20 \mu \mathrm{l} 5 \mathrm{M}$ $\mathrm{NaCl}$ and incubated at $65^{\circ} \mathrm{C}$ overnight. $10 \mu \mathrm{L} 0.5 \mathrm{M}$ EDTA, $40 \mu \mathrm{L} 1 \mathrm{M}$ Tris- $\mathrm{HCl}$ (pH 6.8), and $2 \mu \mathrm{L} 10 \mathrm{mg} /$ $\mathrm{mL}$ Proteinase $\mathrm{K}$ were added to each sample, followed by incubation at $45^{\circ} \mathrm{C}$ for $2 \mathrm{~h}$ to digest proteins. After purification with PCR purification kit (Promega), DNA including Input was recovered by $30 \mu \mathrm{L} \mathrm{H}_{2} \mathrm{O}$ and used as templates for $\mathrm{PCR}$ with primers to amplify the HIVEP2 binding site on SLC6A3. Beta-action (ACTB) was included as a negative target control. The PCR condition was $95^{\circ} \mathrm{C}$ for $3 \mathrm{~min}$, then 40 cycles of $95^{\circ} \mathrm{C}$ for $30 \mathrm{~s}, 56^{\circ} \mathrm{C}$ for $30 \mathrm{~s}$ and $72{ }^{\circ} \mathrm{C}$ for $30 \mathrm{~s}$, followed by $72{ }^{\circ} \mathrm{C}$ for $7 \mathrm{~min}$, using PCR Master Mix (Thermo Scientific) in a final volume of $20 \mu \mathrm{L}$, containing $1 \mu \mathrm{L}$ of eluted DNA as the template. For the negative control ACTB, $0.5 \%$ DMSO was added into the PCR reaction which contained $1 \mu \mathrm{L}$ of eluted DNA as the template. PCR products were resolved by $1.2 \%$ agarose electrophoresis and ethidium bromide-stained bands visualized by the Chemi Doc XRS Molecular Imager. qPCR was used to quantitate the binding activity.

\section{Secondary analysis of dbGaP GWAS datasets}

Three dbGaP GWAS datasets on SUD (all past their embargo periods) and quality control have been reported before $^{13,24}$. Imputation, association tests, logistic regression-based single nucleotide polymorphism (SNP)SNP interactions, and meta-analyses were conducted as reported previously ${ }^{13,24}$. Both secondary data analysis of $\mathrm{dbGaP}$ GWAS datasets and the use of human post mortem brain tissue were approved by McLean Hospital Institutional Review Board (IRB) and/or National Institutes of Health (NIH). 


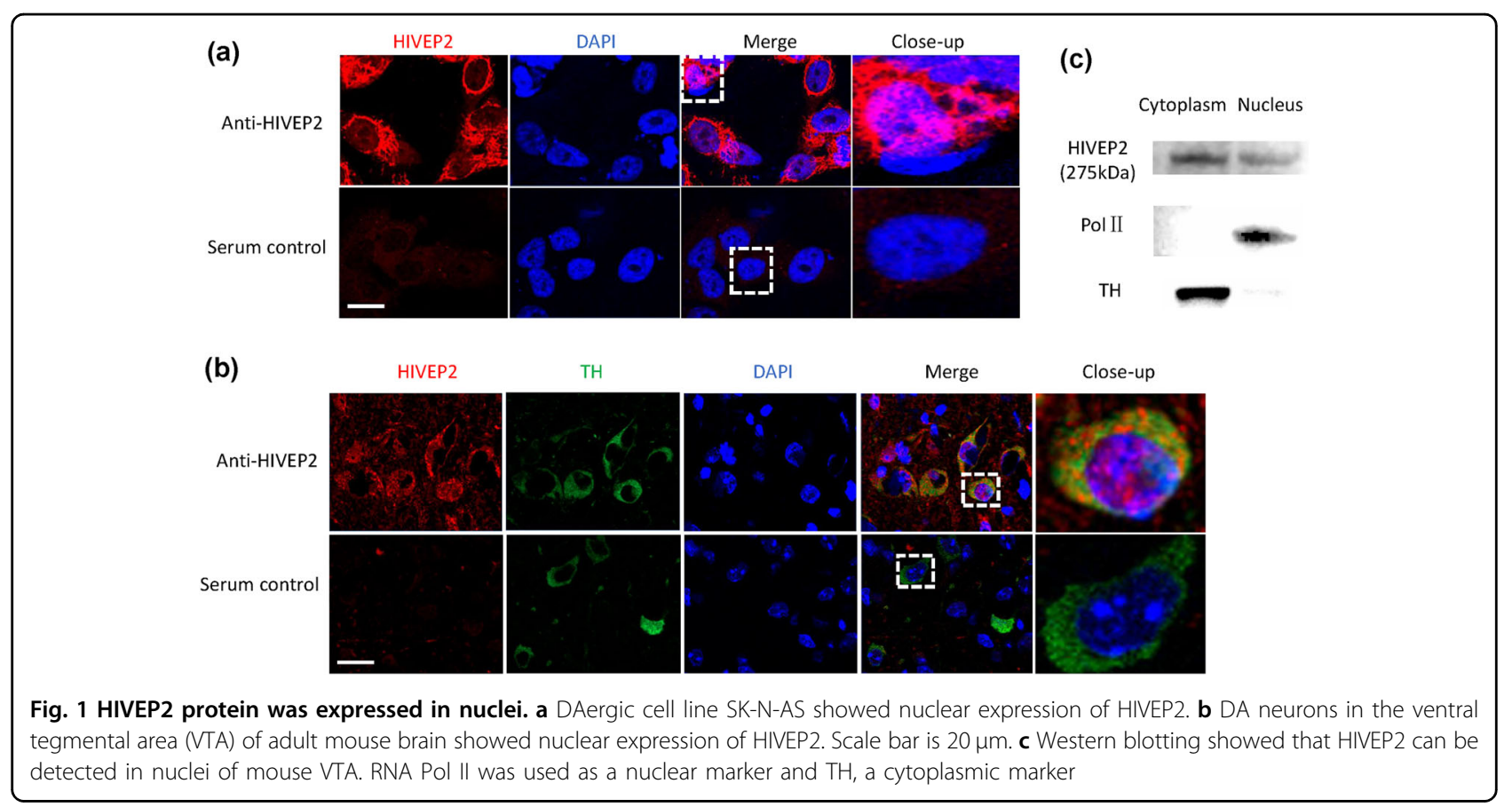

(a)

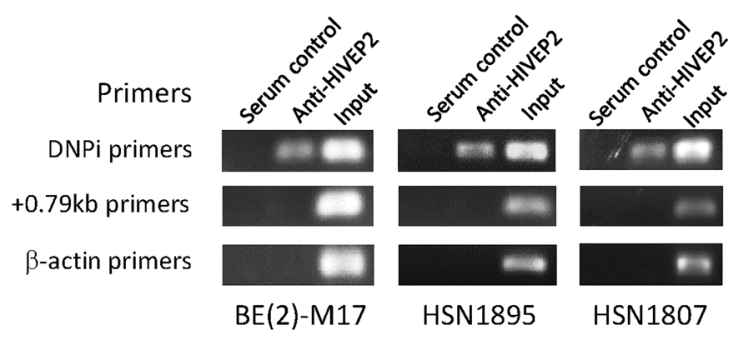

(b)

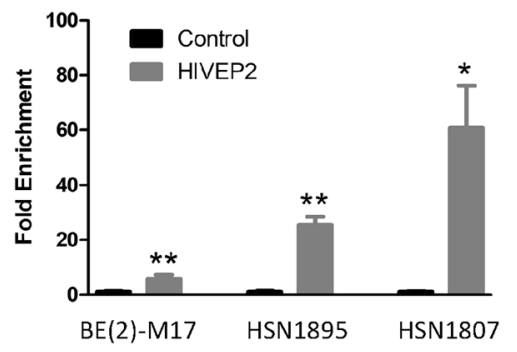

Fig. 2 HIVEP2 bound to the promoter domain of human SLC6A3. a Regular PCR of ChIP showed that anti-HIVEP2 sera can specifically pull down the 121-bp binding domain on Intron 1 of SLC6A3, but neither the $+0.79 \mathrm{~kb}$ locus which was also in Intron 1 and $1 \mathrm{~kb}$ away from 121 -bp nor the ACTB gene. The ChIPs were done on BE(2)-M17 cell line and two human post mortem brain nigral samples: HSN1895 (a 93-year old healthy female) and HSN1807 (a 59-year old healthy male). b Quantification showed fold enrichment by anti-HIVEP2 sera. ${ }^{*} p<0.05 ;{ }^{* *} p<0.01$ (ANOVA Bonferroni post hoc tests, $n=3$ )

\section{Statistical analysis}

Data were all presented as means \pm standard error of mean (SEM) where sample sizes were determined by gene activity stability. Based on GraphPad Prism (v5.04), two-way analysis of variance (ANOVA with Bonferroni post hoc tests) or Student's $t$-test results with $p$ values $<0.05$ were considered as statistically significant.

\section{Results}

By using the 121-bp of haplotype A as bait, many potential clones were obtained. However, based on inframe fusions and adequate expression in DA neurons, HIVEP2 was chosen as a potential DAergic TF for further characterization in this study.

\section{HIVEP2 was present in both cytoplasm and nucleus}

Immunofluorescence staining with anti-HIVEP2 sera localized this protein to both the cytoplasm and nuclei of cultured cells and DA neurons (Fig. 1a, b). In the VTA, most of the protein was expressed in DA neurons. To exclude non-specific reactivity, Western blotting detected the protein, based on its molecular weight, in both cytoplasmic and nuclear fractions of the VTA (Fig. 1c). Greater protein levels were located in the cytoplasm than in the nuclei, as determined by both in vitro and ex vivo assays.

\section{HIVEP2 physically bound to the 121-bp of Intron 1}

Although this protein was cloned as a TF for the 121-bp in yeast cells, the binding activity needed to be verified in 
human cells. To do this three types of samples were used: the neuroblastoma cell line $\mathrm{BE}(2)-\mathrm{M} 17$ of human origin as a cellular model for DA neurons, and post mortem nigral tissue from two healthy subjects, HSN1895, HSN1807. The present results from a ChIP analysis showed that anti-HIVEP2 was able to pull down the 121-bp selectively in all three samples (Fig. 2). This result confirmed the $\mathrm{Y} 1 \mathrm{H}$ result and supported HIVEP2 as a TF in DA neurons.

\section{Overexpression of HIVEP2 upregulated endogenous SLC6A3 activity}

In the human neuroblastoma SK-N-AS cells which served as another model for DA neurons ${ }^{22}$, overexpression of pcDNA3.1 + HIVEP2 increased the HIVEP2 protein levels as determined by Western blotting (Fig. 3a), verifying both the cDNA clone and the anti-HIVEP2 sera. Next, it was determined whether the overexpression could regulate endogenous $S L C 6 A 3$ gene activity in these cellular models. In BE(2)-M17 cells, the overexpression upregulated $S L C 6 A 3$ mRNA levels by $52 \%$ (Fig. 3b). In SK-N-AS cells, the overexpression upregulated SLC6A3 mRNA levels by $30 \%$ (Fig. 3c); and the upregulation went up to $44 \%$ after the cells were treated with hexyl-4-pentynoic acid (HPA), an HDAC inhibitor $^{25}$ (Fig. 3d). In this study, manipulating HIVEP2 expressions had no effect on GAPDH levels, which

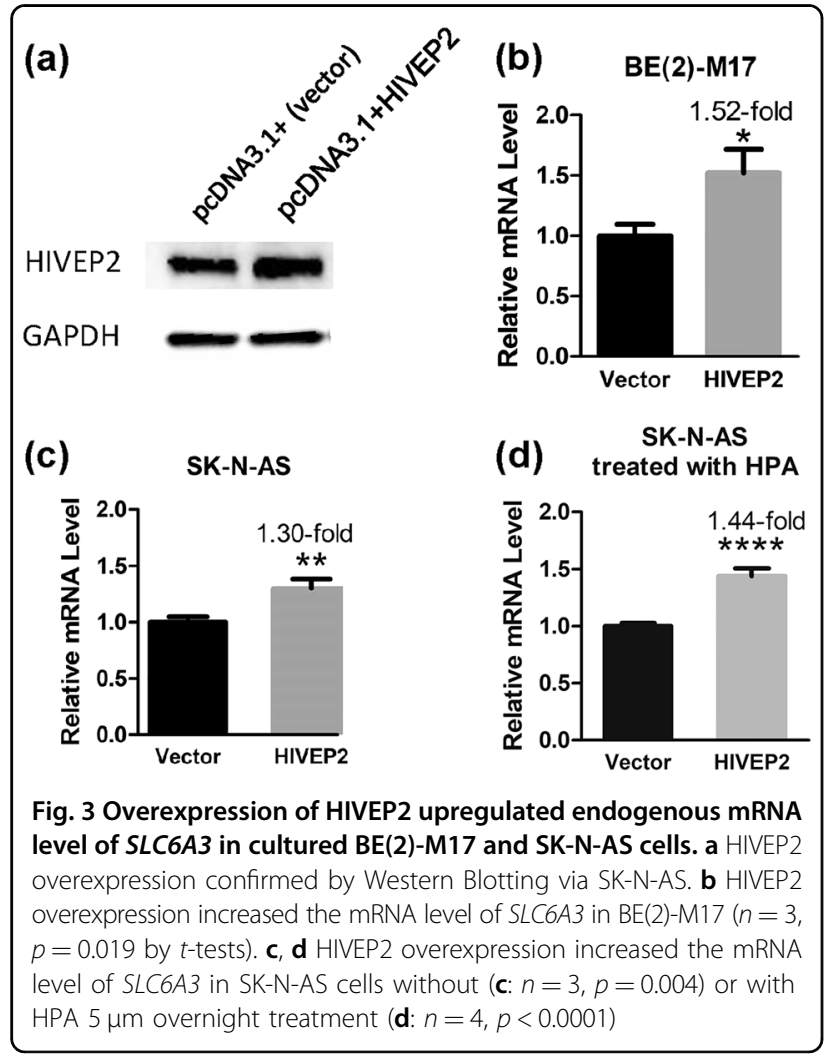

allowed the use of GAPDH as an internal control. These findings indicated that HIVEP2 acted as a transactivator of $S L C 6 A 3$ in these cells, which was consistent with the cloning, the nuclear expression and the 121-bp binding results.

\section{HIVEP2 targeted specific DNA sequences of Intron 1}

A previous study showed that GGGCCTTTCC was the binding motif or consensus sequence (CS) of HIVEP2 $2^{26}$. Two homologous sequences were found in the 121-bp, "1" and "2", which were 54 bp apart from each other. "2" contained two dinucleotide polymorphism (DNPi or rs67175440) alleles ${ }^{13,27}$, A and B, sharing $50-70 \%$ identity with CS (Fig. S1a: "A" defined the haplotype of the bait 121-bp). Luc-reporting analysis showed that overexpression of HIVEP2 could upregulate the SV40 promoter in a "1"- or "2"-dependent manner (Fig. S1b). The results also identified a DsiRNA molecule that could downregulate endogenous HIVEP2 protein expression (Fig. S1c). As a result, this knockdown caused Luc activity reduction to 0.63 -fold via " 1 " as well as to 0.70 -fold via " $2 \mathrm{~A}$ " and " $2 \mathrm{~B}$ ". That is, this HIVEP2 DsiRNA could downregulate the SV40 promoter in a " 1 "- or "2"-dependent manner. These in vitro results suggested that HIVEP2 regulates SLC6A3 activity via binding to GTGGCTTTCT, or to a lesser extent, TTGCCTCTCC or TTGCTCCTCC, documenting the actual sequence(s) in the Intron-1 region bound by HIVEP2.

\section{Sex-dependent alteration of HIVEP2 expression in the $\mathrm{P}$ versus NP rat model}

$\mathrm{P}$ rats display excessive substance (alcohol and other drugs) taking and meet criteria proposed for a valid animal model of SUD. These rats were selected on the basis of their daily free choice intake of $10 \%$ ethanol solution vs. water, and on their preference for the alcohol solution over water. As a consequence, they also show increased responses to some other drugs of abuse ${ }^{28,29}$. To determine potential association between HIVEP2 and SUD, HIVEP2 activity was investigated in the VTA of naive P $v s$ NP rats, an animal model of DAergic pathology ${ }^{30-34}$, using both males and females. The results showed sex-dependence, with activity at both mRNA and protein levels being significantly higher in male $\mathrm{P}$ rats compared with male NP rats, whereas significantly lower in female $\mathrm{P}$ rats compared with female NP rats (Fig. 4). We also have looked at two other recently cloned TFs, SRP54, and Nfe2l1 ${ }^{12}$, and found that neither showed $>50 \%$ changes in the males or any significant changes in the females (protein data not shown here), indicating a selective role of HIVEP2 in the genomic etiology of SUD. These data supported our hypothesis that HIVEP2 can confer a risk to develop SUD, although an important finding was that this effect appeared to be sex-dependent. 


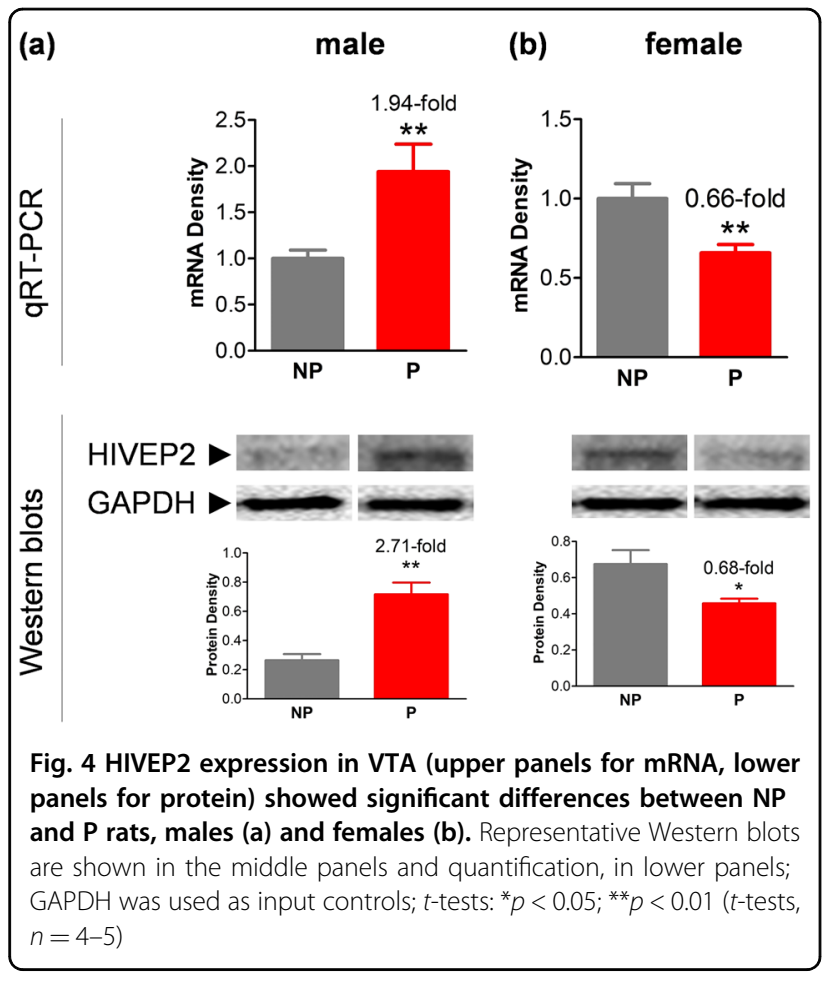

\section{Significant interactions and large effect size epistasis between HIVEP2 and SLC6A3 in SUD}

To validate a clinical relevance of the significant findings in the rat model, we consulted with the dbGaP GWAS datasets from four cohorts with SUD, as described before $^{13}$. Based on meta-analysis of logistic regression case-control association results from the four cohorts of unrelated individuals, we have found that there are $>1200$ significant including more than 300 absolute genome-wide significant interactions between HIVEP2 and SLC6A3 in the genetic etiology of SUD (Fig. 5, top ones detailed in Table S1; "absolute" means Bonferroni correction by all base-pairs of the human genome). These interactions are much more significant than the reported ones between the downregulator's gene ${ }^{\mathrm{AZI} 2} 3^{\prime}$ 'UTR and $S L C 6 A 3^{13}$. For example, rs12525545 in Intron 3 of HIVEP2 interacted with rs748209 located in a distal $(-12 \mathrm{~kb})$ promoter region of $S L C 6 A 3$ with a $p_{\text {meta }}$ value of $1.12 \times 10^{-37}$ and an odds ratio ${ }_{\text {meta }}$ of 0.16 . However, there were no significant main effects, that is, allelic associations between HIVEP2 and SUD (Table S2), with males showing stronger signals than females (details not shown here). There appears to be three rather distinct regions in $S L C 6 A 3$ where the interactions occur but none of previously used markers were located within the two CSs so that the epistatic signals came likely from linkage disequilibrium.

\section{Discussion}

The present study identified HIVEP2, as a new DAergic $\mathrm{TF}$, and has demonstrated that it binds to $S L C 6 A 3$ and regulates $S L C 6 A 3$ activity, which is the hallmark of DAergic neurons. The findings also revealed that altered HIVEP2 activity may be associated with vulnerability for developing SUD; such that altered expression levels were associated with this phenotype in the $\mathrm{P}$ vs NP animal model of SUD (alcohol, nicotine, and cocaine) ${ }^{30-34}$. There seem substantial sex differences in ethanol responses in $\mathrm{P}$ rats. It has been reported that the males show significantly higher preference to $8 \%$ ethanol than the females during a period of 90 days $^{35}$. We have also observed that in an adolescent binge drink model, the males show higher consumption than the females ${ }^{36}$. These behavioral data fit with our finding that elevated HIVEP2 expression in males be a risk factor for AUD in males. Due to limitations of cultured assay systems and of manipulating the large HIVEP2 gene $(10 \mathrm{~kb})$, these in vitro results did not necessarily represent the in vivo effect sizes but the functional human molecular genetic findings may help delineate disease mechanisms in humans.

Previous study showed that the 121-bp of haplotype A, especially a $36 \mathrm{bp}$ subfragment containing the HIVEP2 target " 1 ", displayed inhibitory activity only on the SV40 promoter $^{22}$. It was an expectation that we would be able to clone a trans-repressor, instead of a trans-activator (HIVEP2). Therefore, it remains unknown what mediated the inhibitory activity of 121-bp. One possibility was that the long non-coding RNA (IncRNA) ${ }^{\text {AZI2 }} 3$ 'UTR was contributing to the inhibitory activity. Another possibility was that the activation-based $\mathrm{Y} 1 \mathrm{H}$ system was unable to clone such as a repressor so that other approaches might need to be considered.

HIVEP2 (also known as Schnurri-2) was initially cloned as a zinc finger protein binding to the human immunodeficiency virus type (HIV-1) enhancer and to an enhancer of major histocompatibility complex class I genes $^{26,37}$. This category of TFs are supposed to be expressed primarily in the nuclei but our data suggest that they also are localized to the cytoplasm with unknown function. Lately, this protein has been related to brain function. The gene knockout mice display hyperactivity ${ }^{38}$, consistent with a view that HIVEP2 is an activator of SLC6A3. These mice also display schizophrenia-related phenotypes, increased anxiety, intellectual disability, and memory deficits ${ }^{39-43}$. However, until the present study its activity has not been tested in the context of a vulnerability to develop SUD.

The epistatic association significances were much larger than those for ${ }^{\mathrm{AZI} 2} 3^{\prime} \mathrm{UTR}^{13}$ but it remains unclear where the epistasis signals came from in the meta-analysis of case-control associations with SUD (also alcohol, nicotine 


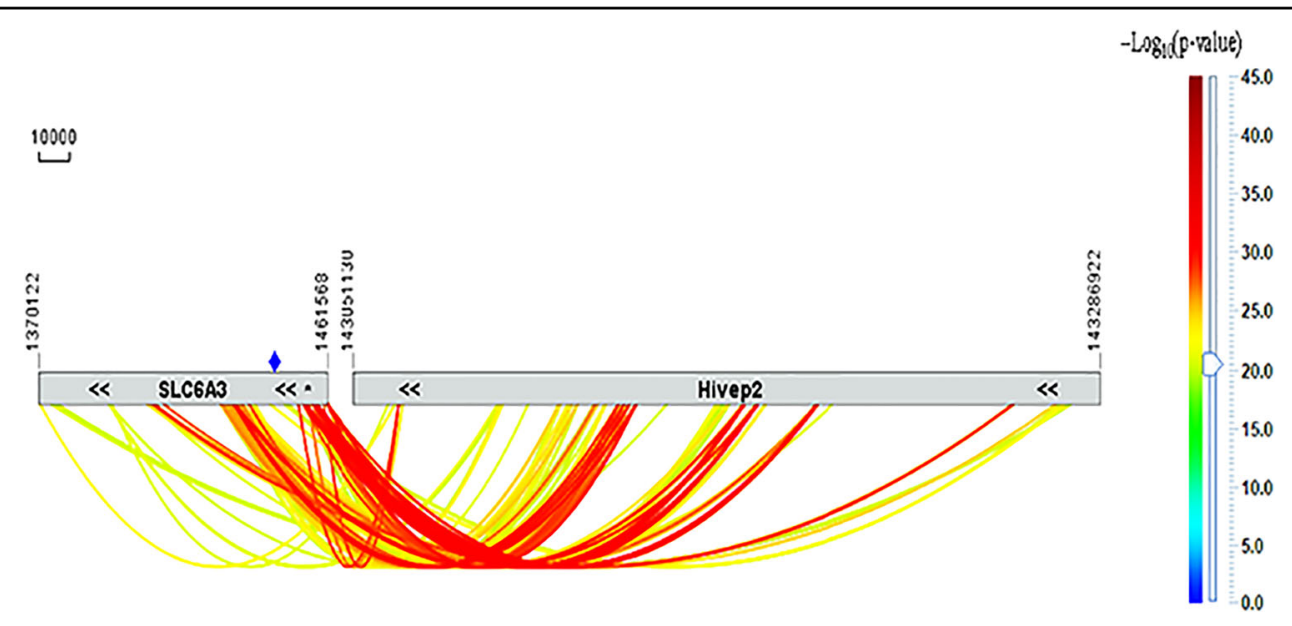

Fig. 5 Human genetics of SUD via SNP interactions (colored curves) between HIVEP2 (on the right) and SLC6A3 (on the left; 121-bp: location indicated by blue diamond). Data are from meta-analysis of logistic regression results by using three dbGaP datasets of SUD (alcohol and cigarette smoking). Gray horizontal bars, on the left is SLC6A3 in chr5 and on the right is HIVEP2 in chr6 with coordinates indicated above the bars; double black arrow symbols, location of transcription start and ending sites (both genes run on the minus strands of the chromosomes); thermometer bar on the right, interaction strength ( $p$-value from meta-analysis) in the form of - $\log _{10}(p$-value); only shown are those reaching absolute genome-wide significance $\left(p<10^{-20}\right)$; chromosomal scale: $10 \mathrm{~kb}$

and cocaine). The HIVEP2 target sequences in the 121-bp carried two known polymorphisms, DNPi (rs2937640 and rs2975223) and rs28382220, both were located in the " 2 " sequences which may have contributed to some of the observed significant epistasis but none of these SNPs were included in the previous GWAS datasets; target sequence "1" carried no known polymorphism. Nevertheless, there was the possibility of "synthetic associations" in the observed associations ${ }^{44,45}$. Based on the epistasis, HIVEP2 could bind to other loci including the distal promoter regions of $S L C 6 A 3$.

A main limitation of this study is that the observed associations do not necessarily indicate causation. To address this, protein-DNA binding activity and associated phenotypes related to SUD could be studied in gene knockout mice. Another unknown is the level of SLC6A3wide, or even genome-wide, binding activity of HIVEP2 in DA neurons. In addition, gene and/or protein activity has not been studied comprehensively in a brain-regionspecific manner. Nor has its function been studied in the cytoplasmic fraction. These limitations warrant further investigations.

\section{Conclusions}

HIVEP2 is a new DAergic TF expressed in both cytoplasm and nuclei. Altered HIVEP2 activity in the VTA is associated with SUD in a rat model; significant HIVEP2SLC6A3 epistasis is found for SUD in humans. These data suggest that regulated HIVEP2 in DA neurons of reward pathways may modulate vulnerability to develop SUD.

\section{Acknowledgements}

We thank Shunsuke Ishii for providing the rabbit anti-HIVEP2 sera, Liangyan Zheng for technical support during the study, and investigators and subjects for contributing to the GWAS, NIH for granting Z.L. with access to the dbGaP GWAS datasets as well as NIDA funding of DA021409 (Z.L.), National Institute on Alcohol Abuse and Alcoholism grants AA015512 and AA13522 (R.L.B.). We are grateful to the Harvard Brain Tissue Resource Center for providing the human brain tissue samples and associated information for these investigations. We thank the International Graduate Exchange Program of Beijing Institute of Technology for supporting J.Z.'s bilateral training at McLean Hospital in U.S.A; C.C. was supported by a Chinese Government Visiting Scholarship.

\section{Author details}

${ }^{1}$ School of Life Science, Beijing Institute of Technology, 100081 Beijing, China. 2Laboratory of Psychiatric Genomics, McLean Hospital, Belmont, MA 02478, USA. ${ }^{3}$ Department of Neurology, the Second Affiliated Hospital of Fujian Medical University, Quanzhou, Fujian Province, P. R. China. ${ }^{4}$ Department of Psychiatry, Institute of Psychiatric Research, Indiana University School of Medicine, Indianapolis, Indiana 46202, USA

\section{Authors' contributions}

Z.L., S.I., R.L.B. and H.Q., study design; J.Z., C.C., Z.L.: experimentation and data analysis; Z.L., writing the manuscript; R.L.B. and J.Z. proof read the manuscript.

\section{Conflict of interest}

The authors declare that they have no conflict of interest.

\section{Publisher's note}

Springer Nature remains neutral with regard to jurisdictional claims in published maps and institutional affiliations.

Supplementary Information accompanies this paper at (https://doi.org/ 10.1038/s41398-019-0573-8).

Received: 9 March 2019 Revised: 30 May 2019 Accepted: 20 June 2019 Published online: 04 October 2019 


\section{References}

1. Wang, Q. et al. Paraquat and MPTP induce neurodegeneration and alteration in the expression profile of microRNAs: the role of transcription factor Nrf2. NPJ Parkinson's. Dis. 3, 31 (2017).

2. Lupu, D. et al. Fluoxetine affects differentiation of midbrain dopaminergic neurons in vitro. Mol. Pharmacol. 94, 1220-1231 (2018)

3. Dang, D. K et al. PKCdelta-dependent p47phox activation mediates methamphetamine-induced dopaminergic neurotoxicity. Free Radic. Biol. Med. 115, 318-337 (2018)

4. Huang, B. et al. Bisphenol a represses dopaminergic neuron differentiation from human embryonic stem cells through downregulating the expression of insulin-like growth factor 1. Mol. Neurobiol. 54, 3798-3812 (2017).

5. Kumar, A. et al. GDNF overexpression from the native locus reveals its role in the nigrostriatal dopaminergic system function. PLoS Genet. 11, e1005710 (2015).

6. Wagenfuhr, L., Meyer, A. K., Marrone, L. \& Storch, A. Oxygen tension within the neurogenic niche regulates dopaminergic neurogenesis in the developing midbrain. Stem cells Dev. 25, 227-238 (2016).

7. Xiong, J. et al. Fenpropathrin, A Widely Used Pesticide, Causes Dopaminergic Degeneration. Mol. Neurobiol. 53, 995-1008 (2016).

8. Bannon, M. J. et al. Decreased expression of the transcription factor NURR1 in dopamine neurons of cocaine abusers. Proc. Natl Acad. Sci. USA 99, 6382-6385 (2002).

9. Wang, J. \& Bannon, M. J. Sp1 and Sp3 activate transcription of the human dopamine transporter gene. J. neurochemistry 93, 474-482 (2005).

10. Jacobs, F. M. et al. Identification of Dlk1, Ptpru and Klhl1 as novel Nurr1 target genes in meso-diencephalic dopamine neurons. Development 136, 2363-2373 (2009).

11. Kanno, K. \& Ishiura, S. Differential effects of the HESR/HEY transcription factor family on dopamine transporter reporter gene expression via variable number of tandem repeats. J. Neurosci. Res. 89, 562-575 (2011).

12. Zhao, Y. et al. Intragenic transcriptional cis-antagonism across SLC6A3. Mol. Neurobiol 56, 4051-4060 (2019).

13. Liu, K. et al. AZI2)3'UTR is a new SLC6A3 downregulator associated with an epistatic protection against substance use disorders. Mol. Neurobiol. 55 5611-5622 (2018).

14. Fuke, S., Sasagawa, N. \& Ishiura, S. Identification and characterization of the Hesr1/Hey1 as a candidate trans-acting factor on gene expression through the $3^{\prime}$ non-coding polymorphic region of the human dopamine transporter (DAT1) gene. J. Biochem. 137, 205-216 (2005).

15. Jankovic, J., Chen, S. \& Le, W. D. The role of Nurr1 in the development of dopaminergic neurons and Parkinson's disease. Prog. Neurobiol. 77, 128-138 (2005).

16. Jimenez-Jimenez, F. J., Garcia-Martin, E., Alonso-Navarro, H. \& Agundez, J. A. PITX3 and risk for Parkinson's disease: a systematic review and meta-analysis. Eur. Neurol. 71, 49-56 (2014).

17. Rouillard, C. et al. Genetic disruption of the nuclear receptor Nur77 (Nr4a1) in rat reduces dopamine cell loss and I-Dopa-induced dyskinesia in experimental Parkinson's disease. Exp. Neurol. 304, 143-153 (2018).

18. Rivetti di Val Cervo, P. et al. Induction of functional dopamine neurons from human astrocytes in vitro and mouse astrocytes in a Parkinson's disease model. Nat. Biotechnol. 35, 444-452 (2017).

19. Chabrat, A. et al. Transcriptional repression of Plxnc1 by Lmx1a and Lmx1b directs topographic dopaminergic circuit formation. Nat. Commun. 8, 933 (2017).

20. Miller, M. L. et al. Ventral striatal regulation of CREM mediates impulsive action and drug addiction vulnerability. Mol. psychiatry 23, 1328-1335 (2018).

21. Stotz, A. \& Linder, P. The ADE2 gene from Saccharomyces cerevisiae: sequence and new vectors. Gene 95, 91-98 (1990).

22. Zhao, Y., Zhou, Y., Xiong, N. \& Lin, Z. Identification of an intronic cis-acting element in the human dopamine transporter gene. Mol. Biol. Rep. 39 5393-5399 (2012)

23. Zhao, Y. et al. Human dopamine transporter gene: differential regulation of 18kb haplotypes. Pharmacogenomics 14, 1481-1494 (2013).
24. Liu, Q. R. et al. Cannabinoid type 2 receptors in dopamine neurons inhibits psychomotor behaviors, alters anxiety, depression and alcohol preference. Sci. Rep. 7, 17410 (2017)

25. Leng, Y., Marinova, Z., Reis-Fernandes, M. A., Nau, H. \& Chuang, D. M. Potent neuroprotective effects of novel structural derivatives of valproic acid: potential roles of HDAC inhibition and HSP70 induction. Neurosci. Lett. 476, 127-132 (2010)

26. Nomura, N. et al. HIV-EP2, a new member of the gene family encoding the human immunodeficiency virus type 1 enhancer-binding protein. Comparison with HIV-EP1/PRDII-BF1/MBP-1. J. Biol. Chem. 266, 8590-8594 (1991).

27. Zhou, Y. et al. Ventral midbrain correlation between genetic variation and expression of the dopamine transporter gene in cocaine-abusing versus nonabusing subjects. Addiction Biol. 19, 122-131 (2014).

28. Bell, R. L. et al. Rat animal models for screening medications to treat alcoho use disorders. Neuropharmacology 122, 201-243 (2017).

29. McBride, W. J., Rodd, Z. A., Bell, R. L., Lumeng, L. \& Li, T. K. The alcohol-preferring (P) and high-alcohol-drinking (HAD) rats - Animal models of alcoholism. Alcohol 48, 209-215 (2014).

30. Gordon, T. L., Meehan, S. M. \& Schechter, M. D. P and NP rats respond differently to the discriminative stimulus effects of nicotine. Pharmacol., Biochem., Behav. 45, 305-308 (1993).

31. Gordon, T. L., Meehan, S. M. \& Schechter, M. D. Differential effects of nicotine but not cathinone on motor activity of $\mathrm{P}$ and NP rats. Pharmacol., Biochem., Behav. 44, 657-659 (1993).

32. Le, A. D. et al. Increased vulnerability to nicotine self-administration and relapse in alcohol-naive offspring of rats selectively bred for high alcohol intake. J. Neurosci. 26, 1872-1879 (2006).

33. Stewart, R. B. \& Li, T. K. The neurobiology of alcoholism in genetically selected rat models. Alcohol Health Res. World 21, 169-176 (1997).

34. Bell, R. L., Rodd, Z. A., Lumeng, L., Murphy, J. M. \& McBride, W. J. The alcoholpreferring $P$ rat and animal models of excessive alcohol drinking. Addiction Biol. 11, 270-288 (2006).

35. Moore, C. F. \& Lynch, W. J. Alcohol preferring (P) rats as a model for examining sex differences in alcohol use disorder and its treatment. Pharmacol., Biochem., Behav. 132, 1-9 (2015).

36. Bell, R. L. et al. Modeling binge-like ethanol drinking by peri-adolescent and adult P rats. Pharmacol., Biochem., Behav. 100, 90-97 (2011).

37. van 't Veer, L. J., Lutz, P. M., Isselbacher, K. J. \& Bernards, R. Structure and expression of major histocompatibility complex-binding protein 2, a 275-kDa zinc finger protein that binds to an enhancer of major histocompatibility complex class I genes. Proc. Natl Acad. Sci. USA 89, 8971-8975 (1992).

38. Takagi, T. et al. Schnurri-2 mutant mice are hypersensitive to stress and hyperactive. Brain Res. 1108, 88-97 (2006).

39. Takao, K. et al. Deficiency of schnurri-2, an MHC enhancer binding protein induces mild chronic inflammation in the brain and confers molecular, neuronal, and behavioral phenotypes related to schizophrenia. Neuropsychopharmacology 38, 1409-1425 (2013).

40. Srivastava, S. et al. Loss-of-function variants in HIVEP2 are a cause of intellectual disability. Eur. J. Hum. Genet. 24, 556-561 (2016).

41. Nakao, A. et al. Immature morphological properties in subcellular-scale structures in the dentate gyrus of Schnurri-2 knockout mice: a model for schizophrenia and intellectual disability. Mol. Brain 10, 60 (2017).

42. Kobayashi, K, Takagi, T., Ishii, S., Suzuki, H. \& Miyakawa, T. Attenuated bidirectional short-term synaptic plasticity in the dentate gyrus of Schnurri-2 knockout mice, a model of schizophrenia. Mol. Brain 11, 56 (2018).

43. Steinfeld, $H$. et al. Mutations in HIVEP2 are associated with developmental delay, intellectual disability, and dysmorphic features. Neurogenetics 17, 159-164 (2016).

44. Kent, J. W. Jr. Rare variants, common markers: synthetic association and beyond. Genet. Epidemiol. 35(Suppl 1), S80-S84 (2011).

45. Takeuchi, F., Kobayashi, S., Ogihara, T., Fujioka, A. \& Kato, N. Detection of common single nucleotide polymorphisms synthesizing quantitative trait association of rarer causal variants. Genome Res. 21, 1122-1130 (2011). 